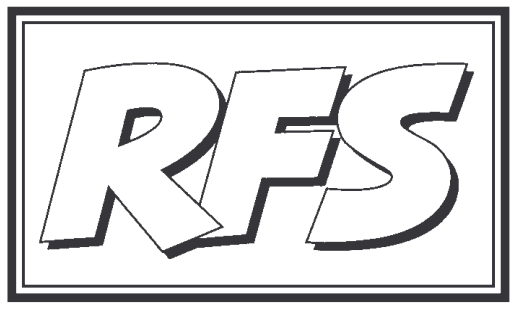

Revista de Fomento Social, 61 (2006), 15-24

\title{
Economía y ética
}

Román PERPIÑÁ GRAU

(PALABRAS CLAVE ÉTICA ECONÓMICA, BENEFICIO, POLITIICA ECONÓMICA, ACTIVIDAD ECONÓMICA

KEY WORDS: ECONOMIC ETHICS, PROFIT, ECONOMIC POLICY, ECONOMIC ACTIVITY)

1 Revista de Fomento Social, n 1, enero-marzo 1946, pp. 15-24. El autor se firma "Consejero de Economía Nacional". 
n aquel histórico primer número de nuestra Revista de Fomento Social - aparecía una valiosa colaboración del maestro de economistas Román Perpiñá Grau.

Había nacido en Reus (1902). Se licenció en Ciencias Económicas en la Universidad de Deusto de Bilbao y obtuvo el título de Intendente Mercantil por la Escuela Superior de Comercio.

La Junta de Ampliación de Estudios le otorgó una beca y posteriormente viajó a Alemania. Allí, bajo la dirección del profesor Liedman, redactó su tesis doctoral, investigación de los fenómenos de concentración de empresas:Sociedades de promoción de empresas en Alemania (1929). Tras una larga colaboración con el profesor Harms, estudió cuestiones ligadas con la localización de actividades económicas y, fundamentalmente, con el proceso de estructuración de toda la actividad económica.

Perpiñá fundó el primer servicio de estudios económicos de España: el Se minario de Estudios Económicos de CHADE, que colaboró con Cambó.

Más tarde se estableció en Valencia, fundó y dirigió el Centro de Estudios Económicos Valencianos desde 1930. Allí concibió y redactó su ensayo más destacado, "De economía hispana" (revista "Weltwirtsches Archiv", 1935, reeditado por Ariel en 1972).

Tras la Guerra Civil, Perpiñá se estableció en Madrid como Consejero de Economía Nacional y se dedicó a realizar varios estudios sobre las posesiones españolas en el Golfo de Guinea.

Maestro de innumerables estudiosos de la economía en América. Román Perpiñá fue profesor en la Universidad Complutense de Madrid y en la Pontificia de Salamanca, siendo el maestro de destacados economistas españoles: Fuentes Quintana, Velarde Fuentes, Tamames, entre otros.

Tenía gran formación en lenguas clásicas, lo que le permitió trabajar con tex tos de grandes autores griegos y latinos con un altísimo rigor, para entender las problemáticas del comportamiento de los pueblos así como el comportamiento económico. Fue autor de numerosas e importantes obras.

Fue condecorado con el premio Príncipe de Asturias de Ciencias Sociales en su primera edición de 1981.

Ya anciano declaró en alguna ocasión que le desagradaba "el sentido económico del mundo, en el que imperan el placer y el dinero”... Murió en 1991.

En este artículo aparecido en el primer número de la RFS en 1946 trata un tema que entonces calificaba "de palpitante actualidad". Sorprende constatar que todavía hoy lo es. El P. Azpiazu, al que se refiere al comienzo el autor, es precisamente el fundador y primer director de la RFS.

\section{RFS}


Muchos hombres de negocios dicen que no; muchos economistas dicen que lo económico no está ni enfrente ni dentro de la ética, sino al lado, en lugar neutro.

A estas posiciones inhibitorias cabe preguntar: ¿Existe un "hombre de negocios" y un "hombre de familia" distinto?, o bien, ¿existe una "vida económica" distinta de la "vida en la sociedad"?; en una palabra, ¿andan por el mundo mezclados, pero separados, los "homines oeconomici", los "homines politici", "homines patres", "homines filii", "homines fratres"?, o ¿es que el hombre obra distintamente en el negocio, en la sociedad, como padre, como hijo o como hermano?

De otra parte, si el hombre obra con distintos principios según sean sus actividades, ¿es que estos principios son contradictorios en bien o en mal de sí mismo o de la sociedad?

No son vanas estas preguntas, pero, además, son necesarias para quien tenga una responsabilidad por ejercer una autoridad y por ejecutar actos que tengan repercusión en una zona, en una actividad, de las que dependan no sólo el éxito de su gestión personal, sino el bienestar de algún grupo social (empresario, profesión liberal, político, etc.).

El tema, viejo de siempre, como vieja es la naturaleza humana y la vida social, no sólo es hoy de palpitante actualidad, sino que ha sido tratado recentísimamente por la autoridad y la competencia de un hombre formado en las más vastas disciplinas como el P. Azpiazu².

No obstante, la obra del P. Azpiazu, por lo completa, presenta tal cantidad de sugerencias, que nos sentimos llamados a hacernos eco de su gran altavoz para discurrir también sobre el problema, no ya como moralistas, sino como economistas, y con ello mostraremos que los conceptos éticos y los econó micos no pueden excluirse, como no se excluye cualquier disciplina, puesto que cada una de ellas no es más que una parte de una única Verdad.

$$
* * *
$$

No queremos, sin embargo, partir de principios preestablecidos y deseamos colocarnos en una posición tal como si no aspiráramos o no deseáramos una determinada conclusión. Es decir, ni con la posición o mentalidad de

2 Véase su obra: La moral del hombre de negocios por Joaquín Azpiazu, S. J., Madrid, Editorial Razón y Fe, 1945. 
un escéptico, para el cual el encogerse de hombros y el arquear de labios hacia abajo es toda la única y perenne contestación a cuanto ve, le sucede o le viene al pensamiento, incluso en sus soledades; ni tampoco en la posición del vulgar creyente que solamente enjuicia sus actos y los de los demás sacándose del bolsillo su regla (la de su partido, la de "sus" principios) y que acepta o rechaza, sin más, con observar si coinciden con "su" medida o le resultan inadecuados en más o en menos. Pero, al mismo tiempo, nuestra posición de interrogante tendrá una previa determinación: el aceptar las conclusiones que de nuestra observación y de su síntesis hallemos, luego de habernos contestado nuestra inquisitoria, así balanceados y con plena indiferencia nuestra espíritu, hasta tanto no vea la verdad que con este equilibrio busca.

Digamos ante todo: ¡Vamos a ver! Ver es mucho más que mirar y expresa ya la representación de un fenómeno en nuestro interior, generalmente producto de nuestra capacidad de observación y de "asombrarnos" de lo que hay o sucede a nuestro alrededor, para captarlo, para comprenderlo. Ver es, pues, el primer momento de nuestra observación consciente inquisidora.

¿Qué vemos, qué distinguimos en el obrar de los hombres y hasta en el de nosotros mismos?

Si tal hacemos, observaremos tres formas principales de actuación en la vida social:

Una de ellas se caracteriza por la fuerza (y, equiparada con ella, el engaño), tanto en las relaciones entre individuos cuanto entre grupos sociales e incluso nacionales. La educación suaviza muchas veces las formas, pero el fenómeno siempre responde a tal principio. Y esta forma aparece no sólo en su ejercicio, sino en los actos conducentes a adquirirla. Es una tendencia tan antigua como el hombre, que, a pesar de haber llegado la Humanidad a poder aceptar como un hecho la conquista de un "alto grado de civiliza ción”, persiste aún en toda su primitivez y se halla en el corazón de muchos hombres, tengan las manos callosas o las puedan comparar con las más estilizadas realizaciones de los príncipes o los más antiguos burgueses que posaron para Hobein, Rafael, Tiziano, Van Dyck o para los retratistas fran ceses de la sociedad de los Luises de la Francia de fines del XVII y del XVIII. $\mathrm{El}$ aprovecharse de la prepotencia, sea del dinero, sea de las armas o de las letras, es indudablemente una de las formas de actuación del hombre, reflejo evidente de una forma de pensar. La aparición, desde la antigüedad, de la posición monopolística y sus manifestaciones en la vida económica moderna 
son una prueba de ello. El hombre ha tendido a eliminar, "sea como sea", a sus competidores, y una vez que ha adquirido una posición de fuerza ha actuado, "sea como sea", para mantenerla y para obtener con ello la máxima satisfacción de sus deseos: riquezas, honores, pasiones. Cuando el hombre, para ello, ha necesitado tener colaboradores, los ha buscado, ha pactado y su grupo ha obrado, a veces, con mucha mayor rigurosidad que lo hizo el hombre aislado, para obtener y para explotar una posición de fuerza, de prepotencia. ¿Siempre? No. No siempre el hombre y el grupo prepotente ha abusado de su fuerza. Lo que observamos es que el abuso de la fuerza constituye "una" de las formas de actuación del hombre en relación con los demás. Y que es típica, es decir, que es tan caracterizada, que permite indi vidualizarla y tenerla por representativa de "una" de las principales formas de obrar, es evidente.

Existe otra forma de actuación que a muchos parecerá reducida a muy corto número de personas y que constituye el revés de la medalla, el ángulo opuesto a la primera forma. Es la forma de actuación de læenerosidad, de la caridad o de la filantropía, como se la llama principalmente en el extranjero. Si la prepotencia tiende a beneficiarse a sí misma y encima a beneficiarse con lo que corresponde a los demás, perjudicándolos, la generosidad, en su límite opuesto, llega a desear para el prójimo todo el beneficio, mediante, incluso, el sacrificio propio. Que esta forma de obrar tiene en la sociedad un campo muy grande de actuación, capaz de poderla considerar también como "una" forma de obrar típica en la sociedad, veremos también que es evidente. En primer lugar observemos la forma de obrar de las madres y de los padres para con sus hijos y, muchas veces, de los hijos para con sus padres. Padres e hijos son miembros de la sociedad, y en sus relaciones existen innumerables actos económicos. Lo mismo es evidente entre esposo y esposa, y los actos de verdadera amistad son, por fortuna, también corrientes en nuestra sociedad. De otra parte, en todos los países la Iglesia, o las iglesias, están en gran parte sostenidas directamente por las dádivas de los fieles, y tanto sus instituciones como las instituciones benéficas que en número mayor que se puede sospechar existen, reciben sus medios de dádivas múltiples y de mandas legales, legados, beneficios, fundaciones de vivos y de difuntos. Y la labor de los ministros de la Iglesia, así como sus Órdenes, junto con la labor de las personas dedicadas a las obras caritativas o filantrópicas (incluso, en gran parte, de la enseñanza, cultura y arte), tiene un valor en la economía, siempre superior a los emolumentos que perciben, si perciben alguno. De consiguiente, la posición interior que hace actuar de manera que no sólo 
no se obtenga beneficio, sino que el propio sacrificio sea el beneficio de los demás, no sólo existe, sino que es típica, característica de "un" obrar muy amplio del hombre y de la sociedad.

Hemos observado el obrar del hombre en dos campos, muy anchos por cierto, pero tan radicalmente opuestos, como opuestos serían los obrares y los pensamientos que los informaron: del "hombre lobo para el hombre", y del hombre a lo San Francisco de Asís. Pero al señalarlos como típicos hemos querido decir que tales obrares no los hemos considerado en esas extremadas actuaciones sino como líneas centrales de dos zonas, y de con siguiente, cabe representarnos a la vez, a derecha e izquierda de cada línea, una atenuación de sendos obrares, y del otro lado, un recrudecimiento de sus características hasta dichos opuestos límites. Si tanto atenuásemos la preponderancia de la fuerza y la preponderancia de la generosidad, podemos concebir que se confundan. Pero más verídica es la observación que en medio de este acercamiento existe otra zona cuya característica central y típica constituye una tercera representación de la actuación del hombre.

Al quedar así, como anuladas y contrapesadas, la fuerza y la generosidad, observamos la real existencia de esta tercera gran zona de equilibrio, en la cual, para que exista relación humana, debemos de observar la existencia de otro fenómeno de actuación que, a la vez que lo rompa para establecer la relación, lo restablezca de nuevo.

Observemos que tal fenómeno existía ya en las dos anteriores: el beneficio, producto de toda relación humana. En la primera el beneficio quedaba todo para el prepotente; en la segunda el sacrificio lo soportaba todo el donante. ¿No es evidente que ese beneficio que existe siempre en la relación humana pueda quedar dividido entre las dos partes de la relación, y que si existe sacrificio también quede compartido?

Hay una palabra a la cual le hemos dado, desde hace ya siglos, una interpre tación común que no corresponde a la que evidentemente tuvo en su origen. Recuerdo que hace pocos días, al preguntarle a un joven humanista, que había bien traducido a Virgilio, por la significación de la palabra " comercio", me contesto -seguramente inadvertido, frente a un economista-, que indicaba cambio de mercancías. Pero asintió cuando le presenté mis dudas de si en su origen no indicó " cambio de mercedes". Y, en efecto, esto es comercio: comercio de cariño, comercio de ideas y... comercio de bienes o de mercan cías y servicios; es decir, intercambio, trueque de algo que tenemos y que, aunque queramos y nos cueste algo desprendernos de ello (generalmente

\section{RFS}


por los sacrificios que hemos hecho por adquirirlo, por producirlo), lo tras pasamos "a cambio" de la merced que nos hace otra persona de darnos algo de lo suyo, con similar sacrificio y a la vez merced de los nuestros. He aquí, de consiguiente, una tercera forma humana de actuar, en la cual existe una compensación de sacrificios, a la vez olvidados, por el beneficio que ambas partes reciben. Que es también forma típica, parece lo más evidente.

Si ahora sintetizamos el obrar humano en estas tres formas típicas que hemos "visto", y si seguimos observando los obrares del hombre, conclui remos que cualquiera de ellas encaja, se explica, se comprende en una de las tres formas típicas observadas, con mayor o con menor acercamiento, pero sin que se nos aparezcan en las relaciones humanas otras formas que más nítidamente expresen el conjunto de nuestros obrares.

Hagamos punto y preguntémonos: Estas tres formas del obrar humano, ¿son las tres "económicas"? ¿Deben de considerarse dentro de la Economía como actos económicos? Con ello entramos en un dintel que parece requerir una petitio principii, es decir, una pregunta explicativa antes de traspasarlo, en nuestro lógico camino: el concepto de Economía. Bien; vamos a "ver".

Ningún economista ha dejado de considerar el factor humano en la Economía, y para todos el hombre es sujeto y objeto de la ciencia económica. Los clásicos, desde fines del siglo XVIII hasta Cairnes, en 1874, basan su teoría en la consideración preponderante o exclusiva de la actividad indivi dual: son individualistas, hasta el punto que un profesor español del siglo pasado, Carreras y González, añade a su concepto de Economía, basado en los clásicos, la siguiente explicación: "Si individualista se llama a todo el que atiende ante todo al bien individual, prescindiendo de la sociedad o tomándola como un medio, la Economía política, no tiene por qué negarlo, es una ciencia individualista." Bien... Los economistas históricos, desde Federico List en 1841, hasta Sombart en la actualidad, operan observando el desarrollo de los grupos humanos y, de consiguiente, con todas sus pasiones e ideas; de ahí que "el hombre" califica su economía y, por lo tanto, le tiene igualmente como factor imprescindible. Y los economistas neoclásicos y modernos, desde Gossen (1854) y especialmente desde Menger y Jevons (1871) y Walras (1874), parten precisamente de una posición psicológica en la formación del precio que constituye el núcleo central de su teoría, y, de consiguiente, el estudio de la actuación humana y de sus relaciones es también 
su problema básico, tanto individualmente como luego en los problemas de masa; pero, además, los modernos economistas estudian cada día con mayor interés los problemas de perturbación de los equilibrios parciales y totales que tan claramente habían expresado en un mundo ideal económico, con lo cual forzosamente tienden a buscar soluciones a fenómenos más reales, debidos precisamente a obrares no tan esquematizados como los primeros supuestos y más adecuados a la actuación del hombre y de los grupos so ciales; y cuando son requeridos como político-económicos ya tienen que dejar muchas veces aparte sus abstracciones y hasta se aplican a grupos políticos con programas "heterodoxos de la ciencia económica", como el profesor socialista Laski; con lo cual se llega a la conclusión de que, si bien se concibe una ciencia de pura investigación y laudables esfuerzos de economistas para mantenerse en tal pureza, la sociedad le pide al economista consejo y, convertido en político-económico, cuando cede, no tiene más remedio que aceptar o rechazar, aunque sea objetivamente, direcciones de actuación política. Y este quedarse al margen de lo político y social no es más que una inteligente posición del economista puro, que sabe que la sociedad, como no obrará solamente en una de las tres formas (para el economista puro, la tercera), sus planes corren el riesgo, sin su culpa, de convertirse, tarde o pronto, en fracaso.

Pues bien; si el hombre es objeto y sujeto en todas las escuelas de econo mistas, a mayor abundamiento no puede prescindirse del hombre cuando se trate la Política económica, sea de la empresa, sea del Estado.

¡Ah!, se dirá; pero el economista sólo estudia los actos económicos de la tercera forma, y en ella, cuando el hombre obra estrictamente y racional mente. Concedido.

Pero ahora cabe preguntarse: ¿Y las perturbaciones económicas? ¿Y las actuaciones de agrupaciones económicas y políticas? ¿Y las actuaciones del Estado? Esto indica claramente que el hombre no obra siempre con una misma forma de actuar, y si ello influye en la empresa o en el complejo económico, ello ha de constituir forzosamente o un dato de la ciencia (que, en efecto, hoy ya mucha recoge) o un dato de la Política económica, que indudablemente no es una disciplina que se pueda desligar de la teoría económica, antes bien, es propiamente su razón de ser la que clama a la ciencia para que le dé razón de sus fenómenos o medios para sus fines. 
De consiguiente, sin necesitar adscribirnos a un concepto o escuela de Economía, observamos que todas las formas de actuación humana influyen en la vida económica, y, por lo tanto, la influencia la podemos observar con mayor claridad sistematizadas en esas formas típicas expuestas.

Si la primera y la tercera -la fuerza y el "comerciar"- nos parecen evidentes como actuaciones en la vida económica, quizá dudemos de la segunda.

Sólo ese dudar de su inclusión hacía reír a los economistas del siglo pa sado. Hoy es muy diferente, porque el mundo, el mundo tal como es, ya no se halla -especialmente nuestra Europa- en un período de expansión y de posible prosperidad para todos. Pero incluso, objetivamente, la muestra de la necesaria inclusión de la segunda forma de actuar me parece descubrirla, en sus indicios, inclusive teóricos, en la utilización por los economistas suecos (hace unos quince años) de la estimación valorada de las actividades de las amas de casa y del servicio doméstico para el cálculo de la renta nacional sueca. Pero la prueba más evidente son las dos guerras mundiales de este siglo: cuando se habla, y con razón, de sus causas predominantemente económicas, todo el mundo hace, interior o exteriormente, referencia a la enorme extensión de las formas de actuar por explotación abusiva de pre potencias de empresas, de grupos sociales altos y bajos, y de políticas; en todos los países y a la vez, como contrapeso, de una parte surgen las políticas sociales por los Estados o "generosidad obligada", las instituciones sociales privadas y... el clamor unánime por la actuación de organismos nacionales e internacionales de socorro, de Cruz Roja, de auxilios, ¡de caridad!, como "única"manera de evitar el perecimiento de hambre de millones de seres inocentes, e incluso no inocentes en todos los países, víctimas directas o indirectas de la primera forma. De consiguiente, si el abuso de la fuerza por los que privadamente o públicamente actúan ha conducido a ese enorme clamor por la segunda forma, ésta no es una entelequia, sino que, de haberse empleado a su tiempo, hubiera equilibrado las internas perturbaciones de la primera y..., lo que es cierto y más importante, hubiera producido un am biente propicio a la contención de la ola de actos de fuerza de los hombres en la vida económica, restableciendo un equilibrio alrededor de la normal relación humana de "intercambio de mercedes".

Y ahora nos podemos volver a preguntar: ¿Tiene algo que ver la Economía con la Ética? 
Las tres formas del obrar humano, así, del obrar humano, sin pensar si es "económico" o no, son a la vez las tres "económicas", y deben de considerarse dentro de la Economía porque son también actos económicos.

De consiguiente, si son actos económicos y el hombre es sujeto y fin de la Economía, nos bastará observar si son o no son éticos dichos tres actos humanos, para concluir ante nuestra primera pregunta.

La observación es fácil para quien tenga tan sólo sereno juicio. La utili zación de una prepotencia para adquirir todo el beneficio de una relación humana es evidentemente una acción no sólo falta de ética, sino socialmente condenada como vil. El obrar generosamente es una acción laudable en sí, y si bien no constituye ni puede constituir el común obrar del hombre en lo social y mucho menos en lo económico estricto, la sociedad que posea almas generosas en sus relaciones familiares y públicas no sólo equilibra las zonas de prepotencias abusivas, sino que evita su aparición o su crecimiento y da a la economía el necesario equilibrio social, e incluso económico, de distribución de riqueza, inalcanzable de otro modo. Y, por fin, si las relaciones entre empresarios y trabajadores, entre compradores y vendedores, entre técnicos y otros prestadores de servicios, y entre el Estado (y con ello las ideas de sus grupos dominantes) y los grupos sociales están prendidas por el principio del "comercio" (dación o prestación de mercedes por dación o contraprestación de mercedes), la vida económica se acerca a los postulados de la sana economía, y las perturbaciones quedan sólo para indicar a los hombres que esta vida no será nunca un paraíso, y que Dios sólo es el que tiene en sus manos el destino de los hombres.

El tema queda planteado, y los lectores, con su juicio y cultura, hallarán substancia larga para alimento de su mente, para el bien propio y para el bien de nuestra sociedad. 